Ophthalmologe 2009 · 106:1016-1016

DOI 10.1007/s00347-009-2091-1

(c) Springer Medizin Verlag 2009

\section{Julius Springer-Preis für Ophthalmologie 2009}

\author{
Herausragende Originalarbeit aus \\ "Der Ophthalmologe" ausgezeichnet
}

Auf der 107. Jahrestagung der Deutschen Ophthalmologischen Gesellschaft (DOG) im September in Leipzig wurde zum 2. Mal der Julius Springer-Preis für Ophthalmologie verliehen. Die Ehrung ging in diesem Jahr an Herrn Dr. Armin Wolf für seine herausragende Originalarbeit zur intravitrealen Anti-VEGF-Behandlung bei retinalen angiomatösen Proliferationen' ${ }^{1}$. Der Preis ist mit EUR 2500,dotiert.

Der Springer Medizin Verlag rief im Jahr 2008 den Julius Springer-Preis für Ophthalmologie ins Leben, um der engagierten Tätigkeit seiner Autoren Rechnung zu tragen und um gezielt im Bereich der Augenheilkunde einen Beitrag zur Förderung des wissenschaftlichen Nachwuchses zu leisten. Der Preis wird jährlich für eine herausragende wissenschaftliche Arbeit verliehen, die in der Rubrik „Originalien“ in der Zeitschrift „Der Ophthalmologe“ erschienen ist.

Der in diesem Jahr prämierte Beitrag widmet sich einer besonderen Form der neovaskulären Makuladegeneration, der Retinalen Angiomatösen Proliferation (RAP). Die Erkrankung wird seit einiger Zeit mit VEGF-Inhibitoren behandelt, wobei die Effekte dieser Behandlung bisher nur in kleinen Fallserien mit relativ kurzem Behandlungszeitraum untersucht wurden. Ziel der Studie von Wolf et al. war es, erstmals anhand einer größeren Fallzahl die Wirksamkeit der Anti-VEGF-Behandlung bei RAP zu untersuchen. $\mathrm{Zu}$ diesem Zweck wurden die Daten von 82 RAP-Patienten, die mit VEGF-

1 Wolf, D. Kook, T. Kreutzer, A. Gandorfer, C. Haritoglou, A. Kampik, M. Ulbig (2008) Intravitreale Anti-VEGF-Behandlung retinaler angiomatöser Proliferationen.

Ophthalmologe 105: 845-851
Inhibitoren behandelt wurden, ausgewertet. Die Ergebnisse zeigen, dass die Erkrankung mithilfe der Anti-VEGF-Therapie erfolgreich therapiert und die Sehkraft der Patienten signifikant verbessert werden kann. Damit konnten Wolf et al. einen Beleg dafür liefern, dass die Therapie mit Anti-VEGFMedikamenten eine sinnvolle Behandlungsoption bei RAP darstellt. Aufgrund der hohen Rezidivquote in der Nachbeobachtungszeit sind hier allerdings engmaschige Kontrollen notwendig, um eine zeitnahe erneute Behandlung zu gewährleisten.

„Herrn Wolf ist insgesamt bei einer sehr großen Anzahl von Patienten mit RAP eine sehr sorgfältige und differenzierte Analyse der Anti-VEGF-Therapieeffekte gelungen. Die Daten sind von hoher Relevanz für das klinische Management von Patienten mit dieser Sonderform der altersabhängigen Makuladegeneration. Die Aussagekraft der Studie liegt deutlich über anderen bisher veröffentlichen Arbeiten mit sehr viel kleinerer Anzahl von Patienten“ so Prof. Holz (Universitäts-Augenklinik Bonn), Herausgeber von „Der Ophthalmologe“ und Vorsitzender der Jury (Prof. Dr. G.K. Krieglstein, Prof. Dr. T. Reinhard, Prof. Dr. H. Hoerauf), in seiner Laudatio.

Der Verlag gratuliert Herrn Dr. Wolf herzlich zum Julius Springer-Preis für Ophthalmologie 2009 und bedankt sich bei allen Autorinnen und Autoren der Zeitschrift für ihren wertvollen Beitrag.

B. Knapp, Heidelberg

Julius Springer-Preis

für Ophthalmologie 2010

Der 3. Julius Springer-Preis für Ophthalmologie wird 2010 im Rahmen der 108. Jahrestagung der Deutschen Ophthalmologischen Gesellschaft in Berlin verliehen und ist mit 2500,00 EUR dotiert.

Ausgezeichnet wird eine herausragende Originalarbeit, die in Der Ophthalmologe publiziert worden ist.

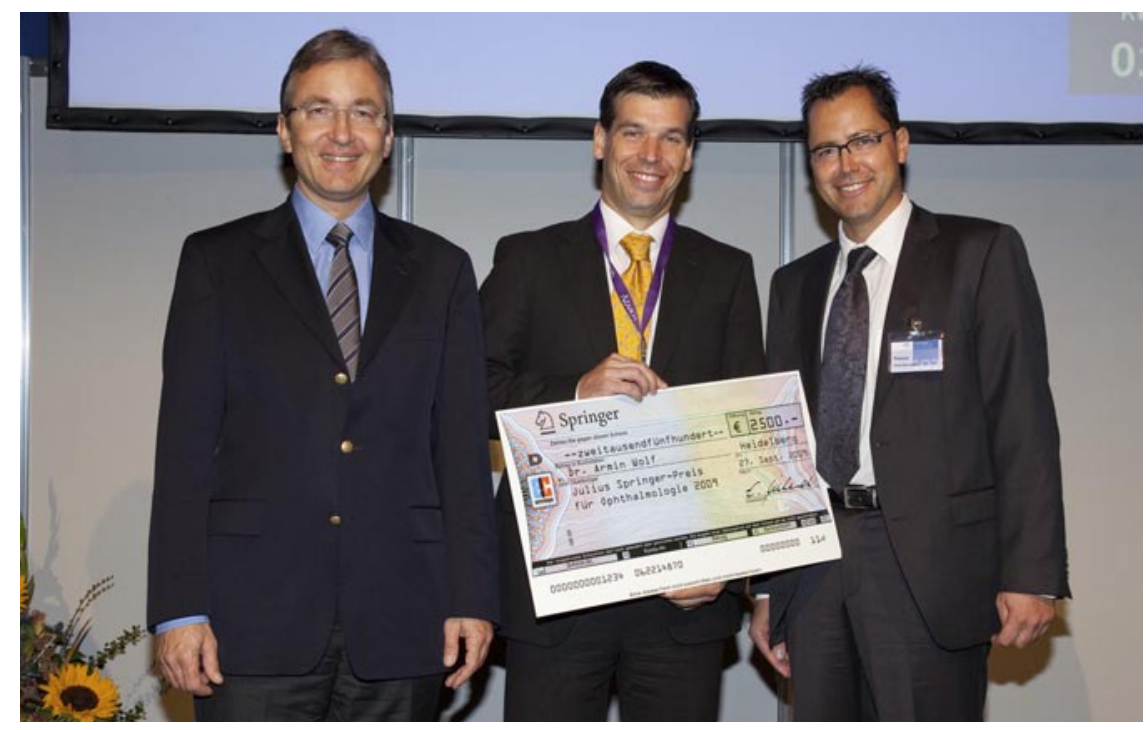

Abb.1 $\triangle$ Preisverleihung

Prof. Dr. F. G. Holz (Herausgeber „Der Ophthalmologe”), Dr. A. Wolf (Preisträger 2009), Dr. P. Herrmann (Leitung Redaktion Fachzeitschriften Medizin/Psychologie, Springer Medizin Verlag) v.l.n.r. 